\title{
The impact of musculoskeletal pain and strenuous work on self-reported physical work ability: a cohort study of Swedish men and women
}

\author{
Kathryn Badarin $^{1} \cdot$. Tomas Hemmingsson ${ }^{1,2} \cdot$ Lena Hillert $^{1,3} \cdot$ Katarina Kjellberg $^{1,3}$
}

Received: 28 June 2021 / Accepted: 1 November 2021 / Published online: 26 November 2021

(c) The Author(s) 2021

\begin{abstract}
Objective We investigated the separate and combined effects of musculoskeletal pain (MSP) and strenuous work (heavy physical workload (PWL)/low-decision authority) on poor physical work ability (WA).

Methods This study uses baseline data from the 2010 Stockholm Public Health Questionnaire (SPHQ) including 9419 workers with good physical WA. Exposure to PWL and decision authority were estimated using sex-specific job-exposure matrices linked to occupations. Exposures (high/low) were combined with the presence of MSP. Follow-up data on physical WA were taken from the 2014 SPHQ and dichotomised (the responses: "moderate", "rather poor" and "very poor" indicated poor WA). Logistic regression models calculated sex-specific odds ratios adjusting for age, education and health and lifestyle factors. Interaction between MSP and strenuous work was examined using the synergy index (SI). Analyses were conducted using SPSS.27.

Results MSP, heavy PWL and low-decision authority were separately associated with poor WA. MSP was associated with higher odds of poor WA than strenuous work for women, the opposite for men. Combinations of MSP and strenuous work often resulted in higher risks of poor WA than when adding the effects of the single exposures (e.g., MSP and heavy PWL men: AOR 4.04 95\% CI 2.00-8.15, women: AOR: 3.25 95\% CI 1.81-5.83). The SI was non-significant for both sexes.

Conclusion Workers with MSP and strenuous work often had higher risks of poor WA than would be expected from adding the effects of the single exposures. To decrease poor WA in this group, strenuous work should be lowered, and MSP addressed in workplaces.
\end{abstract}

Keywords Ergonomics $\cdot$ Musculoskeletal disorders $\cdot$ Work performance $\cdot$ Job-exposure matrix $\cdot$ Epidemiology

\section{Introduction}

Musculoskeletal pain (MSP) is widespread among the European workforce and likely to become more prevalent as the number of older workers increases (EU-OSHA 2020). MSP can restrict individuals' functional capacity and lead to labour market exit (van Rijn et al. 2014). However, many

Kathryn Badarin

kathryn.badarin@ki.se

1 Unit of Occupational Medicine, Institute of Environmental Medicine, Karolinska Institutet, Stockholm, Sweden

2 Department of Public Health Sciences, Stockholm University, Stockholm, Sweden

3 Centre for Occupational and Environmental Medicine, Region Stockholm, Stockholm, Sweden workers with MSP retain good levels of work ability and remain active in the workforce (Pensola et al. 2016). To understand how labour market participation among workers with MSP can be maintained, knowledge of workplace factors associated with work ability for this group is required.

Work ability is a multifaceted concept that encompasses the balance between a workers' physical and psychological functional capacity and the demands of their job (de Zwart et al. 2002). Poor self-reported work ability has high predictive importance for labour market exit (Alavinia et al. 2009; Lundin et al. 2016). Work ability is usually measured using self-report tools of which the 'Work Ability Index' (WAI) is the most common. The WAI is designed to measure different aspects of work ability in relation to work demands, a worker's health status and mental resources (Ilmarinen 2006). 
Multiple individual (e.g., age, obesity, education, leisuretime physical activity and poor musculoskeletal capacity) and work-related factors have been associated with work ability (van Den Berg et al. 2009). Ilmarinen et al. (2005) suggest health and work demands have the largest effect on work ability (Ilmarinen et al. 2005). Specifically, MSP has been associated with poor work ability in several crosssectional (Bayattork et al. 2019; Miranda et al. 2010; Phongamwong and Deema 2015) and longitudinal (Hallman et al. 2019; Tuomi et al. 1991) studies. High physical workload (PWL) has been associated with poor work ability among workers without (Alavinia et al. 2007, van Den Berg et al. 2009) and with MSP (Oliv et al. 2017; Pensola et al. 2016; Skovlund et al. 2020). Psychosocial work factors, such as job control, have been associated with work ability in some studies (Feldt et al. 2009, van Den Berg et al. 2009) but not others (Gamperiene et al. 2008; Pensola et al. 2016; Vries et al. 2013).

A few studies have explored whether the effect of MSP on work ability may differ between workers with and without strenuous work (Bayattork et al. 2019; Nabe-Nielsen et al. 2014; Neupane et al. 2013). A Danish study found associations between increasing intensity of MSP and poor work ability for workers in sedentary and physically active jobs (Bayattork et al. 2019). Slightly greater risks were found among workers with more physically active jobs. A crosssectional study of middle-aged employees found separate associations between MSP or physical job demands and reduced work ability, but an interaction between the exposures on work ability was not found (Nabe-Nielsen et al. 2014). Only one longitudinal study exploring the separate and combined effects of MSP and strenuous work on work ability has been found (Neupane et al. 2013). It reported that MSP and awkward postures were separately associated with poor work ability among food industry employees. However, combined exposure to MSP and awkward postures was not associated with higher risks than when adding the effects of the single exposures. In fact, MSP was associated with a higher risk of poor work ability than strenuous work or the combination of MSP and strenuous work.

Some methodological weaknesses in the aforementioned studies should be noted. First, because most of the studies are cross-sectional (Bayattork et al. 2019; Feldt et al. 2009; Gamperiene et al. 2008; Miranda et al. 2010; Nabe-Nielsen et al. 2014; Oliv et al. 2017; Pensola et al. 2016; Phongamwong and Deema 2015), the ability to make causal interpretations about the separate or combined effects of MSP and strenuous work on poor work ability is limited. Second, the studies used self-reported measures to estimate work-related exposures, which could engender a bias due to self-report, particularly among workers with MSP (Gupta et al. 2018). Last, the effects of exposure to workplace factors on musculoskeletal health can manifest differently among men and women (Arbetsmiljöverket 2013, Fillingim 2000). Yet only two aforementioned studies (Oliv et al. 2017; Tuomi et al. 1991) explored sex-specific associations between MSP or strenuous work and work ability.

In this study, we hypothesised that high PWL or lowdecision authority aggravates the effect of MSP on the risk of poor work ability. Therefore, we investigated the separate and combined effects of MSP and strenuous working conditions [measured using Job-Exposure Matrices (JEMs)] on poor later self-reported physical WA, separately for men and women.

\section{Methods}

\section{Participants and study design}

This study is based on data from the Stockholm Public Health Cohort (SPHC). The SPHC consists of a random sample of people in Stockholm County that responded to repeated questionnaires. Details of the SPHC are described previously (Svensson et al. 2013). The SPHC has multiple sub-cohorts with different baselines starting at 2002. The present study required data on physical work ability which was only available from follow-up questionnaires in 2010 and 2014. Therefore, for this study, respondents to the 2010 questionnaire from two sub-cohorts (2002 and 2006) were combined to create the baseline sample (Fig. 1). Followup data were taken from the 2014 questionnaire. Of the 65,889 respondents to either the $2002(n=31,182)$ or 2006 $(n=34,707)$ baseline questionnaires, 44,494 completed the 2010 questionnaire (this study's baseline) and 32,269 responded to both the 2010 and 2014 questionnaires, an overall response rate of $49 \%$.

Our final sample included employed or self-employed persons likely to not have taken age retirement by 2014 ( $\leq 59$ years old in 2010), with good baseline physical WA, and no missing values for any of the chosen variables ( $n=9419)$ (Fig. 1). Good baseline physical work ability was determined by a response of "very good" or "rather good" the question "How do you rate your current work ability in relation to the physical demands of your job?" from the 2010 questionnaire.

\section{Musculoskeletal pain (exposure)}

A dichotomous variable was created to determine the presence of baseline MSP. A case of MSP was determined by a "yes" response to at least one of three questions in the 2010 SPHC questionnaire: "Have you had any pain in the in the past six months in the (i) upper back or neck, (ii) shoulders or arms or iii) lower back?". Each question had three response categories: "no"; "yes, a few times in the 


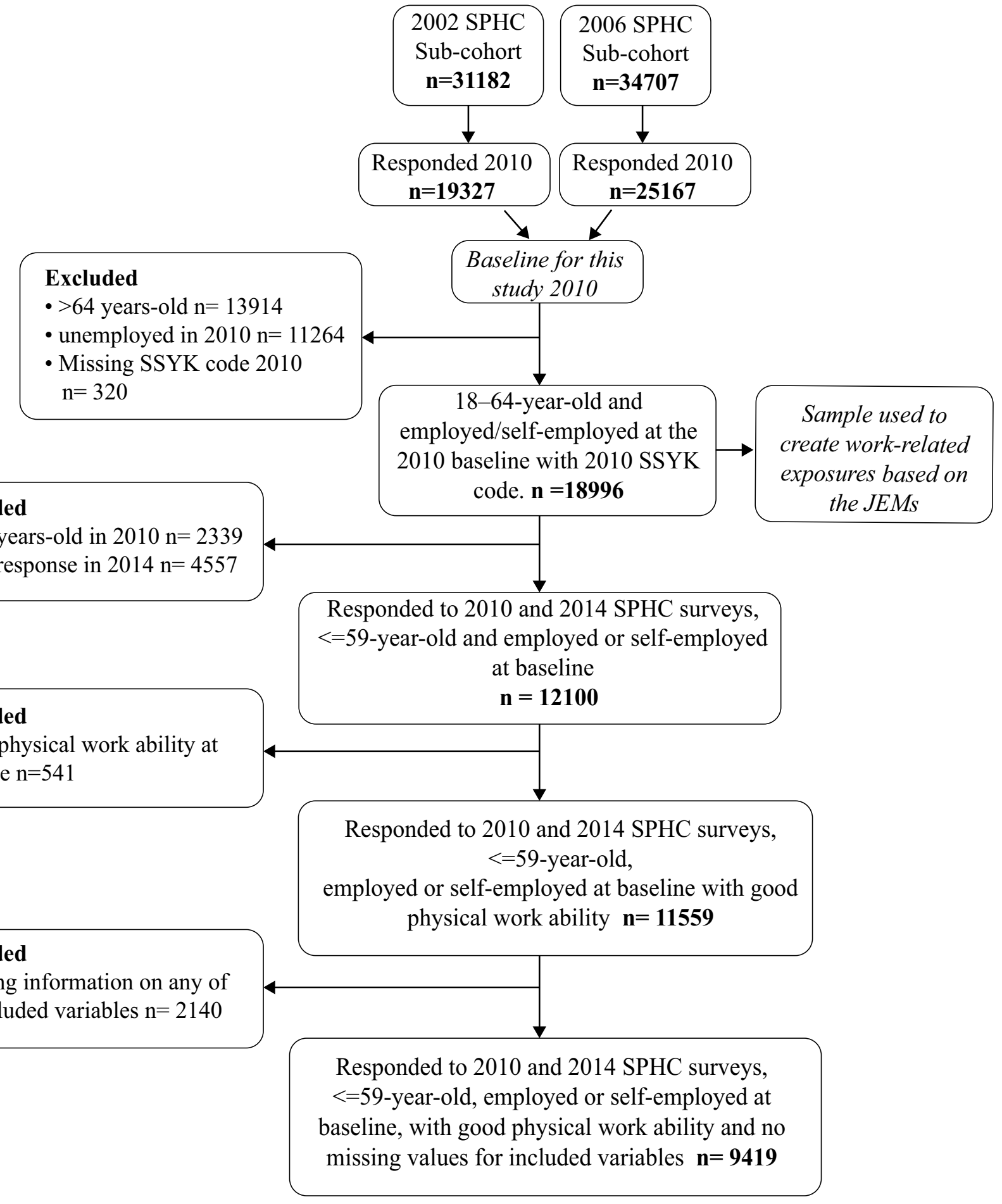

\section{Excluded}

- > 59 years-old in $2010 n=2339$

- Non-response in $2014 \mathrm{n}=4557$

\section{Excluded}

- Poor physical work ability at baseline $\mathrm{n}=541$

\section{Excluded}

- Missing information on any of the included variables $n=2140$

Fig. 1 Sample selection. JEM job-exposure matrix, SPHC Stockholm Public Health Cohort, SSYK Swedish Standard Classification of Occupation

past month or less" or "yes, a few times in the past week or more".

\section{Physical workload (exposure)}

Baseline exposure to PWL was estimated using a Swedish JEM. The construction of the JEM has been described 
previously (Badarin et al. 2021). The JEM was developed using responses to eight questions on physical strenuous work from the biennial Swedish Work Environment Surveys (SWES) between 1997 and 2013. Exposure to eight aspects of PWL (heavy lifting ( $\geq 15 \mathrm{~kg}$ ), physically strenuous work, fast breathing due to PWL, forward bent position, twisted position, working with hands above shoulder level, repetitive work and frequent bending and twisting) were calculated using a 1-to-5/6-point rating scale $(1=$ lowest and 5/6=highest exposure). An index score (overall PWL) was created by summing the scores for each of the eight PWL exposures and calculating a mean value. The JEM provides gender-specific arithmetic mean values for 355 different occupations, coded with the Swedish Standard Classification of Occupation (SSYK) 96 coding system. The SSYK 96 codes (occupational titles) for the 2010 baseline participants were obtained from the Longitudinal Integration Database for Health Insurance and Labor Market Studies (LISA) linked to SPHC.

In this study, the mean JEM values for four PWL exposures; overall PWL, heavy lifting ( $\geq 15 \mathrm{~kg}$ ), working in a forward bent position and fast breathing due to PWL, were assigned to the SSYK codes of all SPHC participants with an SSYK code, 18-64 years old and employed/selfemployed at the 2010 baseline ( $n=18,996)$, before the exclusion of those older than 59 years (Fig. 1). Gender-specific dichotomous variables were created using a median cut-off $(<$ median $=$ low PWL and $\geq$ median $=$ high PWL $)$.

\section{Decision authority (exposure)}

Baseline exposure to decision authority at work was estimated using a Swedish JEM for psychosocial workload. The JEM was developed on the same material and with the same procedure as the physical JEM and has been previously described (Almroth et al. 2021). The JEM provides a gender-specific mean index score for decision authority based on responses to four questions on perceived control over when tasks are conducted, work pace, work breaks and work structure. The JEM scores are linked to occupations using the SSYK 96 coding system. The index scores for decision authority were fixed to 2010 baseline SSYK codes (from LISA) for each participant in this study, based on the same sample of 18,996 workers used for the PWL exposures. Sex-specific binary variables were created using the median as a cut-off ( $>$ median $=$ high decision authority and $\leq$ median $=$ low-decision authority).

\section{Poor self-reported physical work ability (outcome)}

Physical work ability was defined by a single item from the WAI included in the 2014 SPHC questionnaire: "How do you rate your current work ability in relation to the physical demands of your job?" with five responses options: "very good", "rather good", "moderate", "rather poor", and "very poor". Less than "rather good" indicated poor physical work ability. The physical work ability item has shown a strong correlation with the full WAI (Ebener and Hasselhorn 2019), performed well at predicting sick leave (Vingard et al. 2005), and used to explore associations between MSP or strenuous work and work ability in previous studies (Skovlund et al. 2020; Bayattork et al. 2019).

\section{Covariates}

Potential confounders were identified from the literature.

\section{Completed level of education}

Data on completed level of education were taken from LISA. A categorical variable indicated three groups primary ( $\leq 9$ years), secondary (10-12 years) and tertiary ( $>12$ years) education.

The following covariates were from the 2010 SPHC questionnaire.

\section{Psychological distress}

The 12-item General Health Questionnaire (GHQ12) was used to estimate psychological distress. The scores for the GHQ12 range from 0 to 12 . A binary variable was created using $\geq 3$ to signify psychological distress (Banks 1980). The GHQ12 has been shown to be a reliable and valid scoring measure to predict common mental illnesses (Petkovska et al. 2015).

\section{Long term health conditions}

A "yes" response to the question: "Do you suffer from a long-term illness, health problems following an accident, disability or other persistent health problems?" indicated the presence of a long-term health condition.

\section{Body mass index (BMI)}

BMI [weight $(\mathrm{kg}) /($ height $\times$ height $)\left(\mathrm{m}^{2}\right)$ ] was calculated using data from the SPHC. A categorical variable was created with three groups: underweight and normal weight $(\mathrm{BMI}<25)$, overweight $(\mathrm{BMI} \geq 25$ and $\mathrm{BMI}<30)$, and obese $(\mathrm{BMI} \geq 30)$.

\section{Smoking}

Participants were asked "Do you currently smoke daily?" A binary variable yes/no indicated being a current smoker. 


\section{Leisure-time physical activity}

Two questions on leisure-time physical activity were posed. The first question "Average daily amount of walking/cycling over the past 12 months", had the response options: "almost never"; "less than 20 min a day"; "20-40 min a day"; "40-60 min per day"; " $1-1.5 \mathrm{~h}$ per day" or "more than $2 \mathrm{~h}$ a day. The second question "Average weekly amount of physical activity other than walking/cycling" had the response options: "almost never"; "less than $1 \mathrm{~h}$ per week"; " $1-2 \mathrm{~h}$ per week"; "2-3 h per week"; "3-4 h per week"; "4-5 h per week"; "more than $5 \mathrm{~h}$ per week". Responses to both questions were coded as minutes and combined to create an overall estimate of weekly leisure-time physical activity. A final binary variable was created based on the World Health Organization's recommendation of $150 \mathrm{~min}$ of moderateintensity aerobic physical activity a week for adults between ages 18-64 (World Health Organization 2004) ( $\geq 150 \mathrm{~min}$ and $<150$ min weekly leisure-time physical activity).

\section{Statistical analysis}

All statistical analyses were conducted using SPSS version 25.0. First, univariate associations between all covariates and poor work ability were assessed separately for men and women using logistic regression analysis. Second, logistic regression models were used to estimate the associations between combinations of MSP and strenuous work for the risk of poor physical WA, with those jointly unexposed as the reference category: (i) no MSP and non-strenuous work (reference category) (ii) no MSP and strenuous work (iii) MSP and non-strenuous work and (iv) MSP and strenuous work. Crude (OR) and adjusted odds ratios (AOR) were computed for men and women, with $95 \%$ confidence intervals $(95 \% \mathrm{CI})$. Confounding effects of the covariates on the main exposures were explored by grouping covariates according to health and lifestyle factors (model 1), work factors (model 2) and education (model 3). All analyses were adjusted for age. Because educational attainment evidently affects the selection into occupations its inclusion as a confounder could cause over adjustment (34). Therefore, a fully adjusted model excluding education (model 4 ) and a fully adjusted model (model 5) were added. Confounders were chosen based on their empirical and theoretical significance with the exposure and outcome.

Interaction effects between MSP and strenuous work were explored using the synergy index (SI) first presented by Rothman (1986). In this study, the SI measures the extent to which the effect of combined exposure to MSP and heavy PWL or low-decision authority on poor work ability exceeds the sum of the effects of each exposure separately when those unexposed to both exposures are used as reference category (VanderWeele and Knol 2014; Andersson et al. 2005). The SI is defined as:

$$
\mathrm{SI}=\frac{R R_{11}-1}{\left(R R_{10}-1\right)+\left(R R_{01}-1\right)}
$$

If the $\mathrm{SI}>1$ implies there is a synergistic interaction. The 95\% CI for the SI were calculated according to (Andersson et al. (2005).

\section{Results}

Of the 3911 male and 5508 female employees with good work ability in 2010, 161 cases of poor work ability were observed among men and 322 among women after the 4 year follow-up. MSP and more severe levels of MSP were more prevalent among women than men (Appendix 1).

\section{Distribution of covariates in categories of PWL and decision authority}

The proportions of workers above 50 years old, with low education, who smoked daily, were overweight/obese or with less than 150 min of leisure-time physical activity per week was higher among those with high compared to low PWL, for both sexes (Table 1). The opposite was observed for psychological distress.

Among both sexes with low-decision authority, the proportions of workers with low education, who smoked daily, were obese or with $<150$ min of leisure-time physical activity per week was larger than among those with high decision authority. The opposite was observed for psychological distress.

\section{Association between covariates and poor physical work ability}

The univariate analyses showed statistically significant associations between completed education (primary or secondary), long-term health conditions, psychological distress, smoking, being obese and $<150$ min leisure-time physical activity per week and poor work ability for both sexes (Table 2).

\section{Separate and combined effects of MSP and strenuous work on poor physical WA}

Compared to workers without MSP and with non-strenuous work, separate exposure to MSP (e.g., men: OR 2.14 95\% CI: 1.05-4.34, women: OR $3.0195 \%$ CI 1.71-5.28), high PWL (e.g., overall heavy PWL men: OR 3.94, 95\% CI 1.90-8.18, women: OR $2.3995 \%$ CI 1.22-4.65) or 
Table 1 Prevalence of covariates in different PWL and decision authority categories among men and women

\begin{tabular}{|c|c|c|c|c|c|c|c|c|c|c|c|c|}
\hline & Baseline characteristics & & Physi & 1 work & & & Decis & n Autl & rity & & & \\
\hline & & & Low & & High & & High & & Low & & Total & \\
\hline & & & $n$ & $\%$ & $n$ & $\%$ & $n$ & $\%$ & $n$ & $\%$ & $n$ & $\%$ \\
\hline Men & Age & $18-29$ & 67 & 3,1 & 105 & 6,1 & 73 & 3,3 & 99 & 5,9 & 172 & 4,4 \\
\hline 1 & & $30-39$ & 601 & 27,6 & 356 & 20,5 & 572 & 25,6 & 385 & 23,0 & 957 & 24,5 \\
\hline & & $40-49$ & 817 & 37,5 & 633 & 36,5 & 844 & 37,7 & 606 & 36,2 & 1450 & 37,1 \\
\hline & & $50-59$ & 692 & 31,8 & 640 & 36,9 & 748 & 33,4 & 584 & 34,9 & 1332 & 34,1 \\
\hline & Completed Education $^{\mathrm{a}}$ & $>12$ & 1628 & 74,8 & 680 & 39,2 & 1561 & 69,8 & 747 & 44,6 & 2308 & 59,0 \\
\hline & & $10-12$ & 507 & 23,3 & 854 & 49,3 & 592 & 26,5 & 769 & 45,9 & 1361 & 34,8 \\
\hline & & $\leq 9$ & 42 & 1,9 & 200 & 11,5 & 84 & 3,8 & 158 & 9,4 & 242 & 6,2 \\
\hline & Health condition & No & 1732 & 79,6 & 1342 & 77,4 & 1768 & 79,0 & 1306 & 78,0 & 3074 & 78,6 \\
\hline & & Yes & 445 & 20,4 & 392 & 22,6 & 469 & 21,0 & 368 & 22,0 & 837 & 21,4 \\
\hline & Psychological distress $^{\mathrm{b}}$ & No & 1821 & 83,6 & 1514 & 87,3 & 1882 & 84,1 & 1453 & 86,8 & 3335 & 85,3 \\
\hline & & Yes & 356 & 16,4 & 220 & 12,7 & 355 & 15,9 & 221 & 13,2 & 576 & 14,7 \\
\hline & Smoking & No & 2086 & 95,8 & 1571 & 90,6 & 2131 & 95,3 & 1526 & 91,2 & 3657 & 93,5 \\
\hline & & Yes & 91 & 4,2 & 163 & 9,4 & 106 & 4,7 & 148 & 8,8 & 254 & 6,5 \\
\hline & BMI & Underweight/normal & 754 & 34,6 & 477 & 27,5 & 746 & 33,3 & 485 & 29,0 & 1231 & 31,5 \\
\hline & & Overweight & 1239 & 56,9 & 1050 & 60,6 & 1303 & 58,2 & 986 & 58,9 & 2289 & 58,5 \\
\hline & & Obese & 184 & 8,5 & 207 & 11,9 & 188 & 8,4 & 203 & 12,1 & 391 & 10,0 \\
\hline & Leisure physical activity & $\geq 150 \min$ & 1871 & 85,9 & 1380 & 79,6 & 1904 & 85,1 & 1347 & 80,5 & 3251 & 83,1 \\
\hline & & $<150 \min$ & 306 & 14,1 & 354 & 20,4 & 333 & 14,9 & 327 & 19,5 & 660 & 16,9 \\
\hline Women & Age & $18-29$ & 140 & 4,6 & 173 & 7,0 & 151 & 4,9 & 162 & 6,7 & 313 & 5,7 \\
\hline$n=$ & & $30-39$ & 824 & 27,1 & 544 & 22,1 & 822 & 26,5 & 546 & 22,7 & 1368 & 24,8 \\
\hline & & $40-49$ & 1154 & 37,9 & 898 & 36,5 & 1233 & 39,7 & 819 & 34,1 & 2052 & 37,3 \\
\hline & & $50-59$ & 928 & 30,5 & 847 & 34,4 & 900 & 29,0 & 875 & 36,4 & 1775 & 32,2 \\
\hline & Completed Education $^{\mathrm{a}}$ & $>12$ & 2275 & 74,7 & 1190 & 48,3 & 2103 & 67,7 & 1362 & 56,7 & 3465 & 62,9 \\
\hline & & $10-12$ & 708 & 23,2 & 1144 & 46,5 & 906 & 29,2 & 946 & 39,4 & 1852 & 33,6 \\
\hline & & $\leq 9$ & 63 & 2,1 & 128 & 5,2 & 97 & 3,1 & 94 & 3,9 & 191 & 3,5 \\
\hline & Health condition & No & 2393 & 78,6 & 1941 & 78,8 & 2444 & 78,7 & 1890 & 78,7 & 4334 & 78,7 \\
\hline & & Yes & 653 & 21,4 & 521 & 21,2 & 662 & 21,3 & 512 & 21,3 & 1174 & 21,3 \\
\hline & Psychological distress $^{\text {b }}$ & No & 2445 & 80,3 & 2023 & 82,2 & 2500 & 80,5 & 1968 & 81,9 & 4468 & 81,1 \\
\hline & & Yes & 601 & 19,7 & 439 & 17,8 & 606 & 19,5 & 434 & 18,1 & 1040 & 18,9 \\
\hline & Smoking & No & 2867 & 94,1 & 2192 & 89,0 & 2898 & 93,3 & 2161 & 90,0 & 5059 & 91,8 \\
\hline & & Yes & 179 & 5,9 & 270 & 11,0 & 208 & 6,7 & 241 & 10,0 & 449 & 8,2 \\
\hline & BMI & Underweight/normal & 1756 & 57,6 & 1273 & 51,7 & 1776 & 57,2 & 1253 & 52,2 & 3029 & 55,0 \\
\hline & & Overweight & 1059 & 34,8 & 924 & 37,5 & 1084 & 34,9 & 899 & 37,4 & 1983 & 36,0 \\
\hline & & Obese & 231 & 7,6 & 265 & 10,8 & 246 & 7,9 & 250 & 10,4 & 496 & 9,0 \\
\hline & Leisure physical activity & $\geq 150 \mathrm{~min}$ & 2752 & 90,3 & 2161 & 87,8 & 2798 & 90,1 & 2115 & 88,1 & 4913 & 89,2 \\
\hline & & $<150 \min$ & 294 & 9,7 & 301 & 12,2 & 308 & 9,9 & 287 & 11,9 & 595 & 10,8 \\
\hline
\end{tabular}

$P W L$ physical workload

$\stackrel{\text { a }}{ } \geq 12=$ tertiary; $10-12=$ secondary $; \leq 9=$ primary

${ }^{\mathrm{b}}$ Yes $=$ GHQ12 $>3$

low-decision authority (men: OR $1.9895 \%$ CI 1.04-3.78, women: OR 2.47 95\% CI 1.26-4.81) were associated with a statistically significant increased relative risk of poor work ability (Tables 3 and 4).

Separate exposure to high PWL (overall PWL, heavy lifting ( $\geq 15 \mathrm{~kg}$ ), forward bent position or fast breathing) was associated with a larger relative risk of poor work ability than separate exposure to MSP or low-decision authority for men. Among women, MSP was associated with a larger relative risk of poor work ability than heavy PWL or low-decision authority. 
Table 2 Bivariate associations between covariates and poor physical work ability

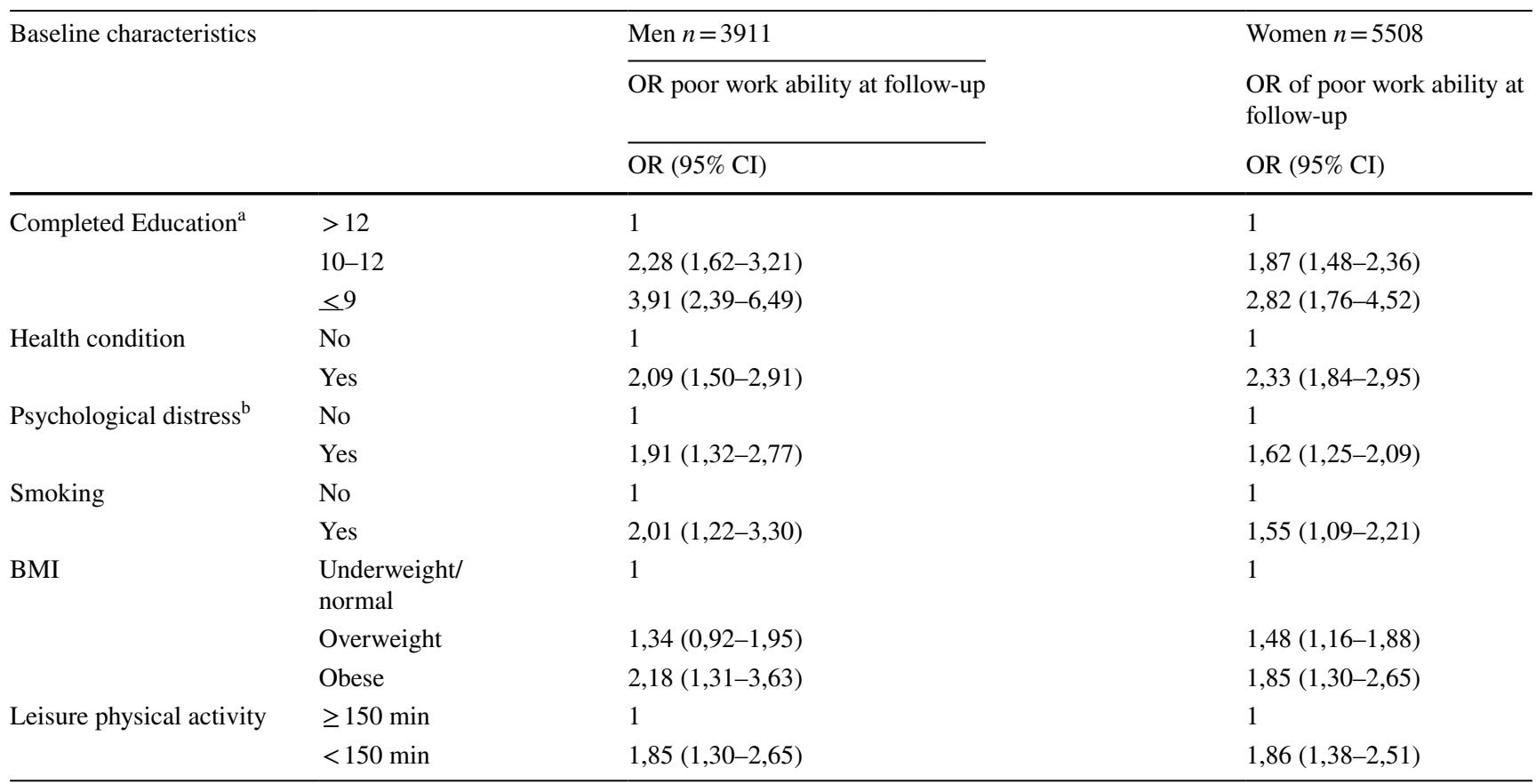

OR odds ratios

95\% CI 95\% confidence intervals

$\stackrel{\text { a }}{ } \geq 12=$ tertiary, $10-12=$ secondary,$\leq 9=$ primary

${ }^{\mathrm{b}}$ Yes $=$ GHQ12 $>3$

Workers with combined exposed to MSP and strenuous work (e.g., overall heavy PWL and MSP, men: OR 6.13 95\% CI, 3.16-11.89, women: OR $6.2095 \%$ CI, $3.57-10.75$ ) had higher risks of poor work ability than when adding the effects of the single exposures (Tables 3 and 4). The $S I$ was non-statistically significant for men (Table 4) but statistically significant for women (e.g., MSP and overall PWL SI $=1.57$ 95\% CI 1.02-2.43) (Table 3).

After adjusting for age, education, smoking, long-term health condition, BMI, psychological distress, leisure-time physical activity and decision authority/heavy PWL, most associations between MSP and poor work ability became non-statistically significant among men [apart from when compared to workers without MSP and not exposed to with physical work causing fast breathing, AOR $2.5895 \% \mathrm{CI}$ 1.20-5.57)]. For women, all associations between MSP and poor work ability remained statistically significant (e.g., AOR 2.48 95\% CI 1.41-4.38).

Separate exposure to all investigated aspects of heavy PWL remained statistically significantly associated with poor work ability for men (e.g., overall heavy PWL men: AOR 2.95 95\% CI 1.38-6.31). For women, statistically significant relative risk of poor work ability remained for heavy lifting (AOR 2.49 95\% CI 1.21-5.16) and fast breathing (AOR 2.28 95\% CI 1.10-4.74). Low decision authority was statistically significantly associated with poor work ability for women (AOR $2.0795 \%$ CI 1.04-4.12), but not men.

All adjusted relative risks for combined exposure to MSP and strenuous work remained statistically significant for both sexes (e.g., MSP and overall heavy PWL men: AOR 4.04 95\% CI 2.00-8.15 women: 3.25 95\% CI 1.81-5.83). However, the adjusted estimates for the $S I$ were non-statistically significant.

\section{Discussion}

\section{Summary of the findings}

To our knowledge, this is the first prospective study to explore the separate and combined effects of MSP and strenuous work (high PWL or low-decision authority) on poor self-reported physical work ability among men and women in the general working population.

MSP, heavy PWL and low-decision authority were separately associated with poor self-reported physical work ability among both sexes. Workers with combined exposure to MSP and heavy PWL or low-decision authority often had higher risks of poor WA than when adding the effects of the single exposures e.g., the SI were often higher than 1. This finding indicates that the relationship between PWL 
Table 3 Crude and adjusted odds ratios (OR with 95 confidence intervals (CI95)) of poor physical work ability at follow-up according to baseline MSP, physical workload/decision authority and their combinations among male workers with good baseline work ability $(n=3911)$

\begin{tabular}{|c|c|c|c|c|c|c|c|c|}
\hline & & Cases/n & Crude & Model 1 & Model 2 & Model 3 & Model 4 & Model 5 \\
\hline \multirow{4}{*}{$\begin{array}{l}\text { MSP/ } \\
\text { heavy } \\
\text { physical work }\end{array}$} & No/no & $10 / 823$ & 1 & 1 & 1 & 1 & 1 & 1 \\
\hline & No/yes & $29 / 595$ & $3,94(1,90-8,18)$ & $3,79(1,82-7,89)$ & $3,37(1,59-7,13)$ & $3,38(1,61-7,09)$ & $3,26(1,54-6,93)$ & $2,95(1,38-6,31)$ \\
\hline & Yes/no & $36 / 1354$ & $2,14(1,05-4,34)$ & $1,86(0,91-3,79)$ & $2,14(1,05-4,33)$ & $2,09(1,03-4,25)$ & $1,87(0,92-3,82)$ & $1,85(0,91-3,77)$ \\
\hline & Yes/yes & $86 / 1139$ & $\begin{array}{c}6,13(3,16- \\
11,89)\end{array}$ & $\begin{array}{c}5,36(2,75- \\
10,47)\end{array}$ & $\begin{array}{c}5,14(2,58- \\
10,24)\end{array}$ & $\begin{array}{c}5,11(2,59- \\
10,08)\end{array}$ & $4,56(2,28-9,11)$ & $4,04(2,00-8,15)$ \\
\hline SI $(95 \% \mathrm{CI})$ & & & $\begin{array}{c}1,35(0,79- \\
2,28)\end{array}$ & $\begin{array}{c}1,30(0,73- \\
2,30)\end{array}$ & $\begin{array}{c}1,26(0,73- \\
2,18)\end{array}$ & $\begin{array}{c}1,27(0,74- \\
2,19)\end{array}$ & $\begin{array}{c}1,22(0,67- \\
2,21)\end{array}$ & $1,16(0,62-2,18)$ \\
\hline \multirow{4}{*}{$\begin{array}{l}\text { MSP/ } \\
\text { heavy } \\
\text { lifting }\end{array}$} & No/no & $11 / 846$ & 1 & 1 & 1 & 1 & 1 & 1 \\
\hline & No/yes & $28 / 572$ & $\begin{array}{c}3,75(1,85- \\
7,62)\end{array}$ & $3,57(1,75-7,29)$ & $3,17(1,54-6,56)$ & $3,18(1,55-6,55)$ & $3,04(1,46-6,32)$ & $2,73(1,30-5,72)$ \\
\hline & Yes/no & $39 / 1380$ & $\begin{array}{c}2,14(1,09- \\
4,20)\end{array}$ & $1,85(0,94-3,66)$ & $2,14(1,09-4,22)$ & $\begin{array}{c}2,09(1,06- \\
4,12)\end{array}$ & $1,87(0,95-3,70)$ & $1,85(0,94-3,66)$ \\
\hline & Yes/yes & $83 / 1113$ & $\begin{array}{c}5,69(3,01- \\
10,76)\end{array}$ & $4,97(2,61-9,47)$ & $4,70(2,43-9,10)$ & $4,71(2,44-9,06)$ & $4,16(2,14-8,09)$ & $3,66(1,86-7,21)$ \\
\hline SI $(95 \% \mathrm{CI})$ & & & $\begin{array}{c}1,29(0,76- \\
2,20)\end{array}$ & $\begin{array}{c}1,26(0,71- \\
2,26)\end{array}$ & $\begin{array}{c}1,19(0,68- \\
2,08)\end{array}$ & $\begin{array}{c}1,21(0,69- \\
2,12)\end{array}$ & $\begin{array}{c}1,16(0,60- \\
2,13)\end{array}$ & $1,10(0,58-2,09)$ \\
\hline \multirow{4}{*}{$\begin{array}{l}\text { MSP/ } \\
\text { forward } \\
\text { bent } \\
\text { position }\end{array}$} & No/no & $11 / 843$ & 1 & 1 & 1 & 1 & 1 & 1 \\
\hline & No/yes & $28 / 575$ & $3,69(1,82-7,49)$ & $\begin{array}{l}3,44(1,68- \\
7,02)\end{array}$ & $3,16(1,51-6,62)$ & $\begin{array}{c}3,16(1,54- \\
6,46)\end{array}$ & $\begin{array}{c}2,95(1,41- \\
6,19)\end{array}$ & $2,69(1,28-5,66)$ \\
\hline & Yes/no & $38 / 1384$ & $2,06(1,04-4,05)$ & $1,79(0,90-3,54)$ & $2,05(1,04-4,05)$ & $\begin{array}{c}1,99(1,01- \\
3,92)\end{array}$ & $\begin{array}{c}1,80(0,91- \\
3,56)\end{array}$ & $1,76(0,89-3,48)$ \\
\hline & Yes/yes & $84 / 1109$ & $\begin{array}{c}5,76(3,04- \\
10,88)\end{array}$ & $4,94(2,60-9,39)$ & $4,87(2,48-9,57)$ & $4,81(2,52-9,21)$ & $\begin{array}{c}4,19(2,13- \\
8,24)\end{array}$ & $3,74(1,89-7,40)$ \\
\hline SI $(95 \% \mathrm{CI})$ & & & $\begin{array}{c}1,33(0,78- \\
2,78)\end{array}$ & $\begin{array}{c}1,28(0,72- \\
2,29)\end{array}$ & $\begin{array}{l}1,25(0,71- \\
2,21)\end{array}$ & $\begin{array}{c}1,27(0,73- \\
2,23)\end{array}$ & $\begin{array}{c}1,16(0,61- \\
2,22)\end{array}$ & $1,16(0,61-2,23)$ \\
\hline MSP/ & No/no & $8 / 868$ & 1 & 1 & 1 & 1 & 1 & 1 \\
\hline \multirow[t]{3}{*}{$\begin{array}{l}\text { Fast } \\
\text { breathing }\end{array}$} & No/yes & $31 / 550$ & $\begin{array}{c}5,99(2,73- \\
13,17)\end{array}$ & $\begin{array}{c}5,51(2,50- \\
12,17)\end{array}$ & $\begin{array}{c}5,27(2,33- \\
11,96)\end{array}$ & $\begin{array}{c}5,12(2,31- \\
11,39)\end{array}$ & $\begin{array}{c}4,84(2,12- \\
11,04)\end{array}$ & $4,32(1,88-9,92)$ \\
\hline & Yes/no & $40 / 1395$ & $\begin{array}{c}3,06(1,42- \\
6,57)\end{array}$ & $\begin{array}{c}2,63(1,22- \\
5,67)\end{array}$ & $\begin{array}{c}3,05(1,42- \\
6,56)\end{array}$ & $\begin{array}{c}2,98(1,39- \\
6,41)\end{array}$ & $\begin{array}{c}2,63(1,22- \\
5,67)\end{array}$ & $2,58(1,20-5,57)$ \\
\hline & Yes/yes & $82 / 1098$ & $\begin{array}{c}7,92(3,81- \\
16,50)\end{array}$ & $\begin{array}{c}6,84(3,27- \\
14,34)\end{array}$ & $\begin{array}{c}6,93(3,21- \\
14,97)\end{array}$ & $\begin{array}{c}6,65(3,15- \\
14,05)\end{array}$ & $\begin{array}{c}5,98(2,75- \\
12,99)\end{array}$ & $\begin{array}{c}5,25(2,39- \\
11,52)\end{array}$ \\
\hline SI $(95 \% \mathrm{CI})$ & & & $\begin{array}{c}1,03(0,66- \\
1,60)\end{array}$ & $\begin{array}{c}1,00(0,63- \\
1,59)\end{array}$ & $\begin{array}{l}0,97(0,33- \\
2,91)\end{array}$ & $\begin{array}{c}0,97(0,33- \\
2,88)\end{array}$ & $\begin{array}{c}0,94(0,58- \\
1,53)\end{array}$ & $0,90(0,54-1,48)$ \\
\hline \multirow{4}{*}{$\begin{array}{l}\text { MSP/low } \\
\text { decision author- } \\
\text { ity }\end{array}$} & No/no & $17 / 838$ & 1 & 1 & 1 & 1 & 1 & 1 \\
\hline & No/yes & $22 / 580$ & $\begin{array}{c}1,98(1,04- \\
3,78)\end{array}$ & $1,80(0,94-3,46)$ & $1,29(0,66-2,51)$ & $1,70(0,89-3,26)$ & $\begin{array}{c}1,20(0,62- \\
2,35)\end{array}$ & $1,16(0,59-2,28)$ \\
\hline & Yes/no & $44 / 1399$ & $\begin{array}{c}1,56(0,88- \\
2,75)\end{array}$ & $1,32(0,75-2,35)$ & $1,57(0,89-2,78)$ & $1,51(0,85-2,66)$ & $1,35(0,76-2,40)$ & $1,33(0,75-2,36)$ \\
\hline & Yes/yes & 78/1094 & $\begin{array}{c}3,59(2,10- \\
6,13)\end{array}$ & $3,08(1,79-5,29)$ & $2,28(1,30-4,00)$ & $2,97(1,72-5,13)$ & $1,99(1,13-3,51)$ & $1,89(1,07-3,34)$ \\
\hline SI $(95 \% \mathrm{CI})$ & & & $\begin{array}{c}1,84(0,78- \\
4,34)\end{array}$ & $\begin{array}{c}1,96(0,68- \\
5,63)\end{array}$ & $\begin{array}{c}1,59(0,61- \\
4,14)\end{array}$ & $\begin{array}{c}1,27(0,74- \\
2,19)\end{array}$ & $\begin{array}{c}1,81(0,36- \\
9,19)\end{array}$ & $\begin{array}{c}1,84(0,30- \\
11,31)\end{array}$ \\
\hline
\end{tabular}

SI synergy index; MSP musculoskeletal pain; all analyses adjusted for age

Model 1: smoking, long-term health condition, BMI, psychological distress and leisure-time physical activity

Model 2: decision authority/ PWL index

Model 3: education

Model 4: model 1+ model 2

Model 5: full model 
Table 4 Crude and adjusted odds ratios (OR with 95 confidence intervals (CI95)) of poor physical work ability at follow-up according to baseline MSP, physical workload/decision authority and their combinations among female workers with good baseline work ability $(n=5508)$

\begin{tabular}{|c|c|c|c|c|c|c|c|c|}
\hline & & Cases $/ n$ & Crude & Model 1 & Model 2 & Model 3 & Model 4 & Model 5 \\
\hline \multirow{4}{*}{$\begin{array}{l}\text { MSP/ } \\
\text { heavy } \\
\text { physical work }\end{array}$} & $\mathrm{No} / \mathrm{no}$ & $14 / 830$ & 1 & 1 & 1 & 1 & 1 & 1 \\
\hline & No/yes & $24 / 605$ & $\begin{array}{c}2,39(1,22- \\
4,65)\end{array}$ & $\begin{array}{c}2,39(1,22- \\
4,68)\end{array}$ & $\begin{array}{c}1,75(0,88- \\
3,46)\end{array}$ & $\begin{array}{c}2,10(1,07- \\
4,11)\end{array}$ & $\begin{array}{c}1,77(0,89- \\
3,51)\end{array}$ & $1,51(0,76-3,02)$ \\
\hline & Yes/no & $107 / 2216$ & $\begin{array}{c}3,01(1,71- \\
5,28)\end{array}$ & $\begin{array}{c}2,58(1,47- \\
4,55)\end{array}$ & $\begin{array}{c}2,99(1,70- \\
5,25)\end{array}$ & $\begin{array}{c}2,94(1,68- \\
5,17)\end{array}$ & $\begin{array}{c}2,56(1,45- \\
4,51)\end{array}$ & $2,48(1,41-4,38)$ \\
\hline & Yes/yes & $177 / 1857$ & $\begin{array}{c}6,20(3,57- \\
10,75)\end{array}$ & $\begin{array}{l}5,25(3,01- \\
9,13)\end{array}$ & $\begin{array}{l}4,50(2,55- \\
7,98)\end{array}$ & $\begin{array}{l}5,40(3,10- \\
9,42)\end{array}$ & $\begin{array}{c}3,83(2,15- \\
6,80)\end{array}$ & $3,25(1,81-5,83)$ \\
\hline SI (95\% CI) & & & $\begin{array}{c}1,57(1,02- \\
2,43)\end{array}$ & $\begin{array}{c}1,48(1,92- \\
2,38)\end{array}$ & $\begin{array}{c}1,33(0,82- \\
2,14)\end{array}$ & $\begin{array}{c}1,49(0,94- \\
2,37)\end{array}$ & $\begin{array}{c}1,26(0,78- \\
2,03)\end{array}$ & $1,18(0,68-2,07)$ \\
\hline \multirow{4}{*}{$\begin{array}{l}\text { MSP/ } \\
\text { heavy } \\
\text { lifting }\end{array}$} & No/no & $11 / 816$ & 1 & 1 & 1 & 1 & 1 & 1 \\
\hline & No/yes & $27 / 619$ & $\begin{array}{c}3,37(1,66- \\
6,85)\end{array}$ & $\begin{array}{c}3,42(1,68- \\
6,97)\end{array}$ & $\begin{array}{c}2,63(1,28- \\
5,41)\end{array}$ & $\begin{array}{c}3,12(1,53- \\
6,35)\end{array}$ & $\begin{array}{c}2,69(1,31- \\
5,56)\end{array}$ & $2,49(1,21-5,16)$ \\
\hline & Yes/no & $97 / 2187$ & $\begin{array}{c}3,48(1,86- \\
6,53)\end{array}$ & $\begin{array}{c}2,99(1,59- \\
5,62)\end{array}$ & $\begin{array}{c}3,44(1,83- \\
6,46)\end{array}$ & $\begin{array}{c}3,42(1,82- \\
6,42)\end{array}$ & $\begin{array}{c}2,95(1,57- \\
5,56)\end{array}$ & $2,87(1,53-5,41)$ \\
\hline & Yes/yes & $187 / 1886$ & $\begin{array}{c}8,20(4,44- \\
15,15)\end{array}$ & $\begin{array}{c}7,00(3,78- \\
12,97)\end{array}$ & $\begin{array}{c}6,38(3,41- \\
11,97)\end{array}$ & $\begin{array}{c}7,44(4,02- \\
13,77)\end{array}$ & $\begin{array}{c}5,48(2,91- \\
10,31)\end{array}$ & $5,02(2,66-9,47)$ \\
\hline SI (95\% CI) & & & $\begin{array}{l}1,51(1,02- \\
2,23)\end{array}$ & $\begin{array}{c}1,40(0,92- \\
2,13)\end{array}$ & $1,35(0,90,2,03)$ & $\begin{array}{c}1,46(0,98- \\
2,16)\end{array}$ & $\begin{array}{c}1,26(0,82- \\
1,95)\end{array}$ & $1,24(0,79-1,94)$ \\
\hline \multirow{4}{*}{$\begin{array}{l}\text { MSP/ } \\
\text { forward } \\
\text { bent } \\
\text { position }\end{array}$} & $\mathrm{No} / \mathrm{no}$ & $13 / 807$ & 1 & 1 & 1 & 1 & 1 & 1 \\
\hline & No/yes & $25 / 628$ & $\begin{array}{c}2,52(1,28- \\
4,97)\end{array}$ & $\begin{array}{c}2,54(1,29- \\
5,02)\end{array}$ & $\begin{array}{c}1,73(0,85- \\
3,54)\end{array}$ & $\begin{array}{c}2,39(1,21- \\
4,71)\end{array}$ & $\begin{array}{c}1,80(0,88- \\
3,69)\end{array}$ & $1,74(0,85-3,57)$ \\
\hline & Yes/no & $103 / 2176$ & $\begin{array}{c}3,09(1,72- \\
5,53)\end{array}$ & $\begin{array}{c}2,60(1,45- \\
4,68)\end{array}$ & $\begin{array}{c}3,04(1,70- \\
5,46)\end{array}$ & $\begin{array}{c}3,00(1,68- \\
5,38)\end{array}$ & $\begin{array}{l}2,56(1,43- \\
4,61)\end{array}$ & $2,47(1,37-4,45)$ \\
\hline & Yes/yes & $181 / 1897$ & $\begin{array}{c}6,53(3,70- \\
11,54)\end{array}$ & $\begin{array}{c}5,60(3,16- \\
9,93)\end{array}$ & $\begin{array}{c}4,49(2,44- \\
8,27)\end{array}$ & $\begin{array}{c}6,07(3,43- \\
10,74)\end{array}$ & $\begin{array}{c}3,96(2,14- \\
7,32)\end{array}$ & $3,79(2,05-7,00)$ \\
\hline SI (95\% CI) & & & $\begin{array}{c}1,57(1,03- \\
2,39)\end{array}$ & $\begin{array}{c}1,52(0,94- \\
2,45)\end{array}$ & $\begin{array}{l}1,30(0,81- \\
2,09)\end{array}$ & $\begin{array}{c}1,55(1,00- \\
2,41)\end{array}$ & $\begin{array}{c}1,33(0,76- \\
2,32)\end{array}$ & $1,26(0,82-1,95)$ \\
\hline \multirow{4}{*}{$\begin{array}{l}\text { MSP/ } \\
\text { Fast breathing }\end{array}$} & $\mathrm{No} / \mathrm{no}$ & $11 / 784$ & 1 & 1 & 1 & 1 & 1 & 1 \\
\hline & No/yes & $27 / 651$ & $\begin{array}{c}3,04(1,50- \\
6,18)\end{array}$ & $\begin{array}{c}3,08(1,51- \\
6,28)\end{array}$ & $\begin{array}{c}2,31(1,12- \\
4,79)\end{array}$ & $\begin{array}{c}2,89(1,42- \\
5,88)\end{array}$ & $\begin{array}{c}2,37(1,14- \\
4,92)\end{array}$ & $2,28(1,10-4,74)$ \\
\hline & Yes/no & $99 / 2185$ & $\begin{array}{c}3,41(1,82- \\
6,39)\end{array}$ & $\begin{array}{c}2,89(1,54- \\
5,44)\end{array}$ & $\begin{array}{c}3,37(1,80- \\
6,32)\end{array}$ & $\begin{array}{c}3,33(1,78- \\
6,25)\end{array}$ & $\begin{array}{c}2,85(1,51- \\
5,36)\end{array}$ & $2,77(1,47-5,21)$ \\
\hline & Yes/yes & $185 / 1888$ & $\begin{array}{c}7,73(4,18- \\
14,29)\end{array}$ & $\begin{array}{c}6,63(3,57- \\
12,28)\end{array}$ & $\begin{array}{c}5,80(3,07- \\
10,97)\end{array}$ & $\begin{array}{c}7,17(3,87- \\
13,27)\end{array}$ & $\begin{array}{l}5,03(2,65- \\
9,55)\end{array}$ & $4,77(2,51-9,06)$ \\
\hline SI (95\% CI) & & & $\begin{array}{c}1,55(1,05- \\
2,30)\end{array}$ & $\begin{array}{c}1,47(0,95- \\
2,28)\end{array}$ & $\begin{array}{c}1,35(0,88- \\
2,07)\end{array}$ & $\begin{array}{c}1,52(1,01- \\
2,27)\end{array}$ & $\begin{array}{c}1,31(0,83- \\
2,08)\end{array}$ & $1,31(0,81-2,12)$ \\
\hline \multirow{4}{*}{$\begin{array}{l}\text { MSP/low } \\
\text { decision } \\
\text { authority }\end{array}$} & No/no & $14 / 843$ & 1 & 1 & 1 & 1 & 1 & 1 \\
\hline & No/yes & $24 / 592$ & $\begin{array}{c}2,47(1,26- \\
4,81)\end{array}$ & $\begin{array}{c}2,52(1,29- \\
4,92)\end{array}$ & $\begin{array}{c}1,96(0,99- \\
3,88)\end{array}$ & $\begin{array}{c}2,35(1,21- \\
4,59)\end{array}$ & $\begin{array}{l}2,02(1,02- \\
4,01)\end{array}$ & $2,07(1,04-4,12)$ \\
\hline & Yes/no & $105 / 2263$ & $\begin{array}{c}2,93(1,67- \\
5,15)\end{array}$ & $\begin{array}{c}2,54(1,44- \\
4,48)\end{array}$ & $\begin{array}{l}2,91(1,66- \\
5,12)\end{array}$ & $\begin{array}{c}2,87(1,63- \\
5,04)\end{array}$ & $\begin{array}{l}2,53(1,44- \\
4,46)\end{array}$ & $2,47(1,40-4,36)$ \\
\hline & Yes/yes & $179 / 1810$ & $\begin{array}{c}6,51(3,76- \\
11,29)\end{array}$ & $\begin{array}{l}5,54(3,18- \\
9,63)\end{array}$ & $\begin{array}{l}5,14(2,90- \\
9,09)\end{array}$ & $\begin{array}{c}6,07(3,50- \\
10,54)\end{array}$ & $\begin{array}{l}4,40(2,48- \\
7,81)\end{array}$ & $4,48(2,52-7,95)$ \\
\hline SI $(95 \%$ CI) & & & $\begin{array}{c}1,62(1,04- \\
2,51)\end{array}$ & $\begin{array}{c}1,50(0,94- \\
2,42)\end{array}$ & $\begin{array}{c}1,43(0,86- \\
2,37)\end{array}$ & $\begin{array}{c}1,49(0,94- \\
2,37)\end{array}$ & $\begin{array}{c}1,35(0,82- \\
2,23)\end{array}$ & $1,40(0,84-2-33)$ \\
\hline
\end{tabular}

SI synergy index; MSP musculoskeletal pain; all analyses adjusted for age

Model 1: smoking, long-term health condition, BMI, psychological distress and leisure-time physical activity

Model 2: decision authority/PWL index

Model 3: education

Model 4: model 1+ model 2

Model 5: full model 
and MSP on poor work ability was more than additive and shows support for the hypothesis that strenuous work aggravates the effect of MSP on poor work ability. However, the estimates for SI were only statistically significant for women. After adjustments, all associations between MSP and poor work ability remained for women, but only one remained for men. Exposure to heavy PWL remained associated with poor work ability among both sexes, but more consistent associations were found among men. Separate exposure to low-decision authority was only associated with poor work ability among women. The relative risks for the combined effects of MSP and strenuous work on poor work ability remained greater than the sum of the individual effects, but the estimates for the SI were not statistically significant.

\section{Comparison with previous studies}

Our finding that separate exposure to MSP was associated with an increased risk of poor self-reported work ability is in accordance with the results of several existing studies (Bayattork et al. 2019; Miranda et al. 2010; Phongamwong and Deema 2015; Hallman et al. 2019; Tuomi et al. 1991), most of them cross-sectional (Bayattork et al. 2019; Miranda et al. 2010; Phongamwong and Deema 2015). Our findings strengthen the current evidence by showing a prospective association between MSP and poor physical work ability.

Our findings also support the results from previous studies showing associations between heavy PWL and work ability for workers without (Alavinia et al. 2007, van Den Berg et al. 2009) and with MSP (Oliv et al. 2017; Pensola et al. 2016; Skovlund et al. 2020). We found stronger associations between heavy PWL and poor work ability among men than women. Only a few existing studies have explored sex-specific associations between PWL and work ability. A Swedish study on workers with neck pain observed stronger associations between low exposure to physical work demands (lifting, twisted work posture, working with hands in shoulder level or higher, and forward bending) and excellent work ability for men than women (Oliv et al. 2017). A Dutch study on male construction workers found associations between awkward postures or manual handling tasks and poor work ability (Alavinia et al. 2007), whereas a Norwegian study on female workers did not observe an association between self-reported level of overall strenuous work and poor work ability (Gamperiene et al. 2008). In fact, like our results, the Norwegian study showed stronger associations between poor self-reported physical health and poor work ability than strenuous work conditions. Overall, our findings and the results of the aforementioned studies allude to a potential difference in the relationship between PWL and poor work ability for men and women.

In this study, low-decision authority was associated with poor physical work ability among women, but not among men. Earlier studies exploring job control and work ability have shown discordant results, some finding an association (Feldt et al. 2009, van Den Berg et al. 2009) and some not (Gamperiene et al. 2008; Pensola et al. 2016). Interestingly, in contrast to our results, the aforementioned study on Norwegian female workers did not find associations between job control and poor work ability (Gamperiene et al. 2008). The differing findings may be a result of methodological differences, such as exploration of different samples and varied measures of control.

One earlier prospective study exploring the separate and combined effects of MSP and strenuous work on poor work ability has been found (Neupane et al. 2013). The study of food industry employees showed separate associations between the presence of multisite MSP and exposure to strenuous work (awkward postures) and poor work ability. MSP was associated with a higher risk of poor work ability than strenuous work, which is consistent with our results for women. The study did not conduct sex-stratified analysis, however, $65 \%$ of their sample were women. In contrast to our findings, the study (Neupane et al. 2013) did not find more than additive effects. In fact, single exposure to MSP was associated with a higher relative risk of poor work ability than combined exposure to MSP and strenuous work. A cross-sectional study exploring associations between increasing intensity of MSP and poor work ability found greater risks of poor work ability among workers with physically active jobs compared to sedentary jobs (Bayattork et al. 2019), but the difference between the OR in the two groups was not statistically significant. A cross-sectional study on middle-aged employees did not find an interaction between MSP and strenuous work on poor work ability (Nabe-Nielsen et al. 2014).

\section{Strengths and limitations}

A strength of this study is its longitudinal design. However, response to population-based surveys is often higher among people with advantaged social positions and those with better health (Martikainen et al. 2016). Therefore, nonresponse and attrition bias may have reduced the strength of our estimates and limit our findings generalisability. That said, an exploration of a sample that included non-responders to the 2014 SPHC questionnaire showed few characteristic differences compared to our final sample. The relatively large sample allowed us to conduct sex-stratified analysis and adjust for range of confounding factors. Nevertheless, residual confounding should be considered.

Another strength is the application of the JEMs. The Swedish JEMs have shown good external validity when predicting worsening MSP (Badarin et al. 2021), disability pension (Falkstedt et al. 2021) or diagnosed depression (Almroth et al. 2021). Studies that examine the use of 
existing JEMs, constructed using similar methods, suggest that the exposure estimates provided by the JEMs can be used to identify occupations with higher risks of MSP and other health outcomes (Rijs et al. 2014, Hanvold et al. 2018; Solovieva et al. 2012). The JEMs allowed us to explore multiple workplace exposures, though, it is hard to disentangle their independent effects as many workplace risk factors are interrelated. It is important to also note some limitations of the JEMS. First, they were created using self-reported data, which is generally perceived as a less accurate measure of PWL than technical measurements e.g., accelerometery (Wells et al. 1997). Nevertheless, self-reported exposure measures are frequently used in large epidemiological studies due to low costs and practicalities. Additionally, because the JEMs were constructed using self-reported data collected from a different sample than the one investigated in this study, the JEMs provide a more independent measure of workplace exposures compared to previous studies that have relied on self-reported data obtained from the same persons. Second, the JEMs provide aggregated estimates of workplace exposures, therefore, the heterogeneity of exposures within occupations is lost. This could lead to nondifferential misclassification and an underestimation of the true estimates (Hanvold et al. 2018).

A further limitation is the single baseline exposure measurement, which does not account for exposure changes over time. Additionally, to create enough statistical power to explore combined exposures and produce sex-specific results our definition of MSP was broad. The strength of the effect of MSP on work ability may differ depending on the severity of MSP explored. It should also be noted that the 2010 SPHC did not include questions on MSP in the lower limbs therefore lower limb MSP was not explored in this study.

Work ability is a complex concept to measure, and its operationalisation varies. It is often infeasible to include the full WAI in surveys; therefore, single items (such as the physical work ability applied in this study) that have shown high correlations with the full WAI are used (Ebener and Hasselhorn 2019).

\section{Interpretation of the results}

Our finding that MSP is an important factor contributing to reduced physical WA, either separately or in combination with strenuous work, underscores the need to combat the prevalence of MSP among workers. MSP was associated with a higher risk of reporting poor work ability among women than men. It should be noted that in our sample, MSP was more prevalent among women than men and more women reported severe MSP than men. Biological (e.g., hormonal) and psychosocial differences (e.g., coping strategies and self-efficacy) between men and women have been identified as explanations for sex/gender-based differences in reports of MSP (Sorge and Totsch 2017; Fillingim 2000). Differences in motor control strategies when performing physical work tasks may also cause women to experience more MSP than men (Cid et al. 2019). Overall, these differences suggest that women have a higher vulnerability to MSP than men and could partly explain why we found that MSP had a greater effect on poor work ability among women than men.

Heavy PWL was associated with a larger risk of poor work ability than MSP among men. A Dutch study also found that high physical demands were more strongly associated with work ability than physical health (respiratory/cardiovascular health) among male construction workers (Alavinia et al. 2009). Typically, men are overrepresented in jobs with high exposure to heavy PWL (Arbetsmiljöverket 2013). This was reflected in our sample as men in the high PWL category reported higher levels of PWL than women in the high PWL category. When exposure to heavy PWL is very high it could have a greater impact on people's self-report of poor work ability than health conditions (e.g., MSP).

In this study, male and female workers with combined exposure to MSP and strenuous work often had higher risks of poor work ability than would be expected from adding each exposure (the SI were often higher than 1). That said, the estimates for the synergy index were only statistically significant for the crude results among women. The additive, and signs of more than additive, effects among the combined exposure group concur with the hypothesis that strenuous work aggravates the effect of MSP on the risk of poor work ability. Reducing exposure to strenuous work appears to be a reasonable approach to lower the risk of poor work ability among workers with combined exposure to MSP and strenuous work. Workers with MSP should have the opportunity to receive support to adjust their work (e.g., environmental adaptations, provision of ergonomic equipment or increased autonomy over the organisation of work tasks) to match a reduction in physical capacity.

\section{Conclusion}

Workers with combined exposure to MSP and strenuous work often had higher risks of poor work ability than would be expected from adding the effects of the single exposures. To decrease the level of poor work ability in this group, exposure to strenuous work should be lowered and MSP should be addressed in workplaces.

\section{Appendix 1}

Distribution of MSP and severity of MSP among level of physical workload or decision authority for men and women. 


\begin{tabular}{|c|c|c|c|c|c|c|c|c|c|c|c|c|}
\hline & & & \multicolumn{4}{|c|}{ Physical workload } & \multicolumn{4}{|c|}{ Decision Authority } & \multicolumn{2}{|l|}{ Total } \\
\hline & & & \multicolumn{2}{|l|}{ Low } & \multicolumn{2}{|l|}{ High } & \multicolumn{2}{|l|}{ High } & \multicolumn{2}{|l|}{ Low } & \multirow[b]{2}{*}{$n$} & \multirow[b]{2}{*}{$\%$} \\
\hline & & & $n$ & $\%$ & $n$ & $\%$ & $n$ & $\%$ & $n$ & $\%$ & & \\
\hline \multirow[t]{10}{*}{ Men $n=3911$} & \multirow[t]{2}{*}{ MSP } & No pain & 823 & 37,8 & 595 & 34,3 & 838 & 37,5 & 580 & 34,6 & 1418 & 36,3 \\
\hline & & pain & 1354 & 62,2 & 1139 & 65,7 & 1399 & 62,5 & 1094 & 65,4 & 2493 & 63,7 \\
\hline & \multirow[t]{5}{*}{ MSP location } & No pain & 823 & 37,8 & 595 & 34,3 & 838 & 37,5 & 580 & 34,6 & 1418 & 36,3 \\
\hline & & Low back & 397 & 18,2 & 276 & 15,9 & 400 & 17,9 & 273 & 16,3 & 673 & 17,2 \\
\hline & & $\begin{array}{l}\text { Arms and/or } \\
\text { Shoulder }\end{array}$ & 135 & 6,2 & 112 & 6,5 & 141 & 6,3 & 106 & 6,3 & 247 & 6,3 \\
\hline & & Upper back & 170 & 7,8 & 121 & 7,0 & 165 & 7,4 & 126 & 7,5 & 291 & 7,4 \\
\hline & & Multisite pain & 652 & 29,9 & 630 & 36,3 & 693 & 31,0 & 589 & 35,2 & 1282 & 32,8 \\
\hline & \multirow{3}{*}{$\begin{array}{l}\text { MSP fre- } \\
\text { quency }\end{array}$} & No pain & 823 & 37,8 & 595 & 34,3 & 838 & 37,5 & 580 & 34,6 & 1418 & 36,3 \\
\hline & & Monthly & 1001 & 46,0 & 812 & 46,8 & 1026 & 45,9 & 787 & 47,0 & 1813 & 46,4 \\
\hline & & Weekly & 353 & 16,2 & 327 & 18,9 & 373 & 16,7 & 307 & 18,3 & 680 & 17,4 \\
\hline \multirow[t]{10}{*}{ Women $n=5508$} & \multirow[t]{2}{*}{ MSP } & No pain & 830 & 27,2 & 605 & 24,6 & 843 & 27,1 & 592 & 24,6 & 1435 & 26,1 \\
\hline & & pain & 2216 & 72,8 & 1857 & 75,4 & 2263 & 72,9 & 1810 & 75,4 & 4073 & 73,9 \\
\hline & \multirow[t]{5}{*}{ MSP location } & No pain & 830 & 27,2 & 605 & 24,6 & 843 & 27,1 & 592 & 24,6 & 1435 & 26,1 \\
\hline & & Low back & 315 & 10,3 & 258 & 10,5 & 303 & 9,8 & 270 & 11,2 & 573 & 10,4 \\
\hline & & $\begin{array}{l}\text { Arms and/or } \\
\text { Shoulder }\end{array}$ & 183 & 6,0 & 138 & 5,6 & 189 & 6,1 & 132 & 5,5 & 321 & 5,8 \\
\hline & & Upper back & 309 & 10,1 & 183 & 7,4 & 315 & 10,1 & 177 & 7,4 & 492 & 8,9 \\
\hline & & Multisite pain & 1409 & 46,3 & 1278 & 51,9 & 1456 & 46,9 & 1231 & 51,2 & 2687 & 48,8 \\
\hline & \multirow{3}{*}{$\begin{array}{l}\text { MSP fre- } \\
\text { quency }\end{array}$} & No pain & 830 & 27,2 & 605 & 24,6 & 843 & 27,1 & 592 & 24,6 & 1435 & 26,1 \\
\hline & & Monthly & 1478 & 48,5 & 1196 & 48,6 & 1511 & 48,6 & 1163 & 48,4 & 2674 & 48,5 \\
\hline & & Weekly & 738 & 24,2 & 661 & 26,8 & 752 & 24,2 & 647 & 26,9 & 1399 & 25,4 \\
\hline
\end{tabular}

MSP musculoskeletal pain

Acknowledgements The authors would like to acknowledge Anette Linnersjö who helped with the data ordering and initial data management.

Author contributions All authors conceived the study, designed the analyses, contributed to the interpretations of the results, and reviewed and edited the final manuscript. $\mathrm{KB}$ analysed the data and wrote the paper.

Funding Open access funding provided by Karolinska Institute. This study was financially supported by the Swedish Research Council for Health, Working Life and Welfare (FORTE 2017-02024).

Data availability Data may be obtained from a third party and are not publicly available. The data used for this study were obtained from Statistics Sweden (SCB).

\section{Declarations}

Conflict of interest None declared.

Ethical approval The Regional Ethics Review Board in Stockholm, Sweden, approved the research (Dnr number 2018/225-31). All data were anonymised by Statistics Sweden.

Open Access This article is licensed under a Creative Commons Attribution 4.0 International License, which permits use, sharing, adaptation, distribution and reproduction in any medium or format, as long as you give appropriate credit to the original author(s) and the source, provide a link to the Creative Commons licence, and indicate if changes were made. The images or other third party material in this article are included in the article's Creative Commons licence, unless indicated otherwise in a credit line to the material. If material is not included in the article's Creative Commons licence and your intended use is not permitted by statutory regulation or exceeds the permitted use, you will need to obtain permission directly from the copyright holder. To view a copy of this licence, visit http://creativecommons.org/licenses/by/4.0/.

\section{References}

Alavinia SM, van Duivenbooden C, Burdorf A (2007) Influence of work-related factors and individual characteristics on work ability among Dutch construction workers. Scand J Work Environ Health 33:351-357

Alavinia SM, de Boer AGEM, van Duivenbooden JC, Frings-Sen MHW, Burdorf A (2009) Determinants of work ability and its predictive value for disability. Occup Med (oxford) 59:32-37

Almroth M, Hemmingsson T, Sörberg Wallin A, Kjellberg K, Burström B, Falkstedt D (2021) Psychosocial working conditions and the risk of diagnosed depression: a Swedish register-based study. Psychol Med 1-9. https://doi.org/10.1017/S003329172100060X

Andersson T, Alfredsson L, Källberg H, Zdravkovic S, Ahlbom A (2005) Calculating measures of biological interaction. Eur J Epidemiol 20:575-579

Arbetsmiljöverket (2013) State of knowledge report: physical work, gender, and health in working life [Online]. Stockholm: Swedish Work Environ Auth. https://www.av.se/globalassets/filer/publi 
kationer/kunskapssammanstallningar/physical-work-gender-andhealth-in-working-life-kunskapssammanstallning-2013-9-eng.pdf. Accessed 04 Sep 2020

Badarin K, Hemmingsson T, Hillert L, Kjellberg K (2021) Physical workload and increased frequency of musculoskeletal pain: a cohort study of employed men and women with baseline occasional pain. Occup Environ Med (London, England) 78:558-566

Banks MH, Clegg CW, Jackson PR, Kemp NJ, Stafford EM, Wall TD (1980) The use of the General Health Questionnaire as an indicator of mental health in occupational studies. J Occup Organ Psychol 53:187-194

Bayattork M, Jakobsen MD, Sundstrup E, Seidi F, Bay H, Andersen LL (2019) Musculoskeletal pain in multiple body sites and work ability in the general working population: cross-sectional study among 10,000 wage earners. Scand J Pain 19:131

Cid MM, Oliveira AB, Januario LB, Côté JN, De Fátima Carreira Moreira R, Madeleine P (2019) Are there sex differences in muscle coordination of the upper girdle during a sustained motor task? J Electromyogr Kinesiol 45:1-10

de Zwart BCH, Frings-Dresen MHW, van Duivenbooden JC (2002) Test-retest reliability of the work ability index questionnaire. Occup Med (oxford, England) 52:177

Ebener M, Hasselhorn H (2019) Validation of short measures of work ability for research and employee surveys. Int J Environ Res Public Health 16:3386

Eu-Osha EAFSAHAW (2020) Work-related musculoskeletal disorders: why are they still so prevalent? Evidence from a literature review [Online]. Luxembourg: European Agency for Safety and Health at Work - EU-OSHA. Available: https://euosha.blumm.it/bundl es/app/assets/website/download/Work_related_musculoskeletal_ disorders_why_so_prevalent_report.pdf. Accessed 04 Sep 2020

Falkstedt D, Hemmingsson T, Albin M, Bodin T, Ahlbom A, Selander J, Gustavsson P, Andersson T, Almroth M, Kjellberg K (2021) Disability pensions related to heavy physical workload: a cohort study of middle-aged and older workers in Sweden. Int Arch Occup Environ Health 94:1851-1861

Feldt T, Hyvönen K, Mäkikangas A, Kinnunen U, Kokko K (2009) Development trajectories of Finnish managers' work ability over a 10-year follow-up period. Scand J Work Environ Health 35:37-47

Fillingim RB (2000) Sex, gender, and pain: women and men really are different. Curr Rev Pain 4:24-30

Gamperiene M, Nygård JF, Sandanger I, Lau B, Bruusgaard D (2008) Self-reported work ability of Norwegian women in relation to physical and mental health, and to the work environment. J Occup Med Toxicol (london, England) 3:8-8

Gupta N, Heiden M, Mathiassen S, Holtermann A (2018) Is selfreported time spent sedentary and in physical activity differentially biased by age, gender, body mass index, and low-back pain? Scand J Work Environ Health 44:163-170

Hallman DM, Holtermann A, Dencker-Larsen S, Birk Jørgensen M, Nørregaard Rasmussen CD (2019) Are trajectories of neck-shoulder pain associated with sick leave and work ability in workers? A 1-year prospective study. BMJ Open 9:e022006. https://doi.org/ 10.1136/bmjopen-2018-022006

Hanvold TN, Sterud T, Kristensen P, Mehlum IS (2018) Mechanical and psychosocial work exposures: the construction and evaluation of a gender-specific job exposure matrix (JEM). Scand J Work Environ Health 45:239-247

Ilmarinen J (2006) The work ability index (WAI). Occup Med (oxford) $57: 160-160$

Ilmarinen J, Tuomi K, Seitsamo J (2005) New dimensions of work ability. Int Congr Ser 1280:3-7

Lundin A, Kjellberg K, Leijon O, Punnett L, Hemmingsson T (2016) The association between self-assessed future work ability and long-term sickness absence, disability pension and unemployment in a general working population: a 7-year follow-up study. J Occup Rehabil 26:195-203

Martikainen P, Laaksonen M, Piha K, Lallukka T (2016) Does survey non-response bias the association between occupational social class and health? Scand J Public Health 35:212-215

Miranda H, Kaila-Kangas L, Heliövaara M, Leino-Arjas P, Haukka E, Liira J, Viikari-Juntura E (2010) Musculoskeletal pain at multiple sites and its effects on work ability in a general working population. Occup Environ Med 67:449-455

Nabe-Nielsen K, Thielen K, Nygaard E, Thorsen SV, Diderichsen F (2014) Demand-specific work ability, poor health and working conditions in middle-aged full-time employees. Appl Ergon 45:1174-1180

Neupane S, Virtanen P, Leino-Arjas P, Miranda H, Siukola A, Nygård $\mathrm{CH}$ (2013) Multi-site pain and working conditions as predictors of work ability in a 4-year follow-up among food industry employees. Eur J Pain 17:444-451

Oliv S, Noor A, Gustafsson E, Hagberg M (2017) A lower level of physically demanding work is associated with excellent work ability in men and women with neck pain in different age groups. Saf Health Work 8:356-363

Pensola T, Haukka E, Kaila-Kangas L, Neupane S, Leino-Arjas P (2016) Good work ability despite multisite musculoskeletal pain? A study among occupationally active Finns. Scand J Public Health 44:300-310

Petkovska MS, Bojadziev MI, Stefanovska VV (2015) Reliability, validity and factor structure of the 12-item general health questionnaire among general population. Open Access Maced J Med Sci 3:478-483

Phongamwong C, Deema H (2015) The impact of multi-site musculoskeletal pain on work ability among health care providers. J Occup Med Toxicol (London) 10:21

Rijs KJ, van der Pas S, Geuskens GA, Cozijnsen R, Koppes LLJ, van der Beek AJ, Deeg DJH (2014) Development and validation of a physical and psychosocial job-exposure matrix in older and retired workers. Ann Occup Hyg 58:152-170

Rothman KJ (1986) Modern epidemiology. Little, Borwn and Company, Boston

Skovlund SV, Bláfoss R, Sundstrup E, Andersen LL (2020) Association between physical work demands and work ability in workers with musculoskeletal pain: cross-sectional study. BMC Musculoskelet Disord 21:166

Solovieva S, Mazza M, Pehkonen I, Kausto J, Miranda H, Shiri R, Kauppinen T, Heliövaara M, Burdorf A, Husgafvel-Pursiainen K, Viikari-Juntura E (2012) Development and validation of a job exposure matrix for physical risk factors in low back pain. PLoS ONE 7:e48680

Sorge RE, Totsch SK (2017) Sex differences in pain. J Neurosci Res 95:1271-1281

Svensson AC, Fredlund P, Laflamme L, Hallqvist J, Alfredsson L, Ekbom A, Feychting M, Forsberg B, Pedersen NL, Vågerö D, Magnusson C (2013) Cohort profile: the Stockholm public health cohort. Int J Epidemiol 42:1263-1272

Tuomi K, Luostarinen T, Ilmarinen J, Klockars M (1991) Work load and individual factors affecting work disability among aging municipal employees. Scand J Work Environ Health 17:94-98

Van Den Berg TIJ, Elders LAM, De Zwart BCH, Burdorf A (2009) The effects of work-related and individual factors on the work ability index: a systematic review. Occup Environ Med 66:211-220

van Rijn RM, Robroek SJW, Brouwer S, Burdorf A (2014) Influence of poor health on exit from paid employment: a systematic review. Occup Environ Med 71:295

Vanderweele TJ, Knol MJ (2014) A tutorial on interaction. Epidemiol Methods 3:33-72

Vingard E, Lindberg P, Josephson M, Voss M, Heijbel B, Alfredsson L, Stark S, Nygren A (2005) Long-term sick-listing among women 
in the public sector and its associations with age, social situation, lifestyle, and work factors: a three-year follow-up study. Scand J Public Health 33:370-375

Vries H, Reneman M, Groothoff J, Geertzen J, Brouwer S (2013) Self-reported work ability and work performance in workers with chronic nonspecific musculoskeletal pain. J Occup Rehabil 23:1-10

Wells R, Norman R, Neumann P, Andrews D, Frank J, Shannon H, Kerr M (1997) Assessment of physical work load in epidemiologic studies: common measurement metrics for exposure assessment. Ergonomics 40:51-61
World Health Organization (2004) W. Global strategy on diet, physical activity and health: physical activity and adults [Online]. Geneva World Health Organization, WHO. https://www.who.int/dietp hysicalactivity/factsheet_adults/en/. Accessed 04 Sep 2020

Publisher's Note Springer Nature remains neutral with regard to jurisdictional claims in published maps and institutional affiliations. 